\title{
A Pilot Study for Testing the Efficiency of a Novel Integrated On-line Platform Using Tumoral Biomarkers Evaluation in Colorectal Cancer
}

\author{
ALEXANDRU ISAIC\#, ADELINA CHEVERESAN", AMADEUS DOBRESCU*, CRISTI TARTA ${ }^{1}$, LAURIAN STOICA ${ }^{1}$, \\ CIPRIAN DUTA ${ }^{1}$, LAZAR FULGER ${ }^{1}$ \\ Victor Babes University of Medicine and Pharmacy, Faculty of Medicine, 2 Eftimie Murgu Sq., 300041, Timisoara, Romania
}

\begin{abstract}
Colorectal cancer $(C R C)$ is one of the leading causes of death among cancer patients in Romania. It is known as a heterogeneous disease, characterized by diversion in multiple molecular pathways during its evolutionary process. This research was focusing on the two biomarkers that are related to $C R C$, specifically Carcinoembryonic Antigen (CEA) and Carbohydrate Antigen 19-9 (CA 19-9). Being an integral part of a wider study, we explored the use of a novel platform to support the strictly controlled process of data collection on colorectal cancer cases. We collected data about the patients regarding baseline clinical parameters, medical history, important comorbidities, intraoperative findings and adverse events, tumor characteristics, histological findings and main laboratory data (blood cell count, serum protein electrophoresis, liver and kidney function tests, serum levels of CEA and CA19-9). The results suggest that these two biomarkers are useful preoperative indicators of local recurrences and prognosis in colorectal cancer. Increased levels of both tumormarkers are correlated with an advanced stage of the disease and a poor prognosis. Finally, this study aims to provide the necessary infrastructure, geared towards efficient, safe and comprehensive clinical evaluation of $C R C$ cases.
\end{abstract}

Keywords: colorectal cancer, pilot study, on-line platform, biomarkers

One of the most frequent types of malignancies is colorectal cancer (CRC) being the third most common cancer worldwide, reported by some authors as the most common causes of female cancer mortality followed by lung and/or breast cancer [1]. Additionally, some authors asserted that it is the main cause of cancer death worldwide [2,3]. Around $4.6 \%$ of men ( 1 in 22 ) and $4.2 \%$ of women (1 in 24) will be diagnosed with CRC during lifetime [4]. The risk of colorectal cancer is starting to increase after the age of 40 years; the risk doubles with each subsequent decade and continues to rise exponentially [5].

The prognosis of colorectal cancer is better if the diagnosis is established earlier. Survival after colorectal cancer diagnosis strongly correlates with the stage of the disease, local tumor spreading, lymph node association and the presence of distant metastases. Nevertheless, there remains great inter-patient variability regarding treatment outcome. A combination of biochemical factors, histopathological features, genomic profile, environmental factors and other clinical aspects are capable to influence the prognosis and treatment response. Along with those clinical and paraclinical features, there are some specific biomarkers that predict the severity and the evolution of the disease. CEA level is the most often used tumor marker in colorectal cancer [6]. Its value must be checked before surgery, but also it can be used during therapy to assess individual response to treatment or after therapy to predict an eventual recurrence. An additional blood marker that may be elevated in colorectal cancer is the CA 19-9. It is known that patients with preoperative increased CA 19-9 level had a poorer 5-year survival than patients with preoperative increased CEA levels [7].

The main objective of this research was to elaborate and test the feasibility of an on-line platform utilized to collect the data gathered from the selected cases included in this study. In order to achieve this objective, we focused on the two biomarkers that are related to CRC -CEA and CA 19-9.

\section{Experimental part}

Materials and methods

The study was performed in agreement with the ethical guidelines of the Helsinki Declaration and was approved by the Ethics Committee of our Center. For this study, we included all the cases that underwent surgery for colorectal cancer during the last three years (2014-2017) at the $2^{\text {nd }}$ Department of Surgery, Emergency County Hospital, Timi oara, Romania. Two hundred and ninety-three patients with histologically confirmed CRC and admitted to our Clinic were eligible for participation and initially included in the study. The exclusion criteria were the decline of the patient to share the personal data/medical information, the refuse to write and sign the written informed consent, missing data or skipping scheduled periodic medical control. Consequently, 36 patients were excluded from the study by those reasons. The final study lot was represented by 257 cases which agreed to read and sign the informed consent. Before surgery, the patients were introduced in to the on-line platform by baseline clinical parameters like age, gender, place of residence, addiction to smoking and/or alcohol. Height and weight were measured using standard techniques. The platform provided a field that automatically calculates the Body Mass Index (BMI) using the standard formula (BMI $=\mathrm{kg} / \mathrm{m}^{2}$ ) where kg represents the patient's weight in kilograms and the surface of the body as their height squared. Obesity was defined when the BMI was $\geq 30.00$ [8]. The subsequent data were collected also on the online platform database for each patient: presenting signs and symptoms, preoperatory TNM, surgery (data entered in real time from mobile device by operating room nurse or after the surgery by the surgeon), oncologic radicality of the operation, 
location of the tumor, intraoperative TNM and main laboratory data (blood cell count, serum protein electrophoresis, liver and kidney function tests, and serum levels of CEA and CA19-9). Pathology report containing macroscopic features at presentation, diameters of the tumor, number of lymph nodes harvested and metastatic ones, visceral spreading, number and diameters of distant metastatic lesions, microscopic subtype of the tumor, depth of penetration into the bowel wall, cell differentiation, local or distal invasion were further introduced into the platform. Follow-up with physical examination of the patients and blood samples at 3 and 6 months after the surgery.

For testing the feasibility of this new on-line platform, the authors tested the predictive value of various CEA and CA 19-9 levels both before and after surgery and others parameters from above. For this reason, we selected from the database only the patients that had 3 measurements of CEA and CA 19-9 available at three timeline points: before surgery, at 3 and 6 months after surgery. Serum CEA and CA 19-9 were determined using enzymatic immunoassay kits, with the upper limit of normal defined as $5 \mathrm{ng} / \mathrm{mL}$ for CEA and $38 \mathrm{U} / \mathrm{mL}$ for CA 19-9. For the patients that presented a recurrence in the first year after surgery, we analyzed the correlation between the values of preoperative tumor markers and the values at the time of relapse. The platform utility and feasibility were evaluated by data completeness.

\section{Statistical analysis}

All data were analyzed using a statistical software package, IBM Statistical Package for the Social Sciences (SPSS 17.0). Student t-tests and $\div 2$ analysis were performed in order to compare independent variables in question between each other and depend ones.

\section{Results and discussions}

Some of the most relevant data about the patients for this study are presented in table1. The mean (SD) age of the patients at diagnosis was 62.4 (10.7) years and $52.52 \%$ (135 patients) were male. Most patients had a primary tumor in the colon $(n=224 ; 87.16 \%)$ and metastasis in the liver ( $n=44 ; 17.12 \%)$.

Furthermore, we analyzed the relationship between postoperative and preoperative CA19-9 and CEA values and recurrence sites as shown in table 2 . The percentage of patients with an increased postoperative serum CA19-9 was significantly higher among those who also had a high preoperative value of this biomarker. Fifty-two of the patients, representing $20.23 \%$ of the cases had a recurrence in the first year after surgery. Regarding recurrence site, patients with liver metastases and peritoneal recurrence presented an elevated serum CA19-
Table 1

DEMOGRAPHY OF THE PATIENTS WITH CRC INTRODUCED ON THE PLATFORM; $* n=$ no. OF PATIEN

\begin{tabular}{|c|c|}
\hline Variable & $\begin{array}{c}\text { Mean (SD)/n }(\%) \\
n=257^{\circ} \text { cases }\end{array}$ \\
\hline \multicolumn{2}{|l|}{ Gender } \\
\hline Male & $135(52.52)$ \\
\hline Female & $122(48.37)$ \\
\hline Age at diagnosis (years) & $62.4(10.7)$ \\
\hline Smokers & $186(72.37)$ \\
\hline \multicolumn{2}{|l|}{ Associated pathology } \\
\hline - & $141(54.86)$ \\
\hline NO & $116(45.14)$ \\
\hline \multicolumn{2}{|l|}{ Location primary tumor } \\
\hline Colon & $224(87.16)$ \\
\hline Rectum & $33(12.84)$ \\
\hline \multicolumn{2}{|l|}{ Dukes classification } \\
\hline$B$ & $94(36.57)$ \\
\hline $\mathrm{C}$ & $102(39.68)$ \\
\hline$T=-1$ & $61(23.75)$ \\
\hline \multicolumn{2}{|l|}{ Tumor size (cm) } \\
\hline$<5$ & $68(26.46)$ \\
\hline$\geq 5$ & $189(73.54)$ \\
\hline \multicolumn{2}{|l|}{ Histology } \\
\hline $\begin{array}{l}\text { Well, moderately } \\
\text { Poorly, mucinous }\end{array}$ & $\begin{array}{l}194(7 / 48) \\
53(20.62)\end{array}$ \\
\hline \multicolumn{2}{|l|}{ Metastasis } \\
\hline Liver & $44(17.12)$ \\
\hline Lung & $12(4.66)$ \\
\hline Elsewhere & $21(8.17)$ \\
\hline \multicolumn{2}{|l|}{ Peritoneal dissemination } \\
\hline YES & $54(30.61)$ \\
\hline NO & $203(69.39)$ \\
\hline \multicolumn{2}{|l|}{ Treatment } \\
\hline Surgery & $257(100)$ \\
\hline Chemotherapy & $223(86.77)$ \\
\hline Radiotherapy & $54(21.01)$ \\
\hline Recurrence & $52(20.23)$ \\
\hline
\end{tabular}

9. The proportion of patients with elevated postoperative CA19-9 was significantly increased among those who presented liver metastases. Additionally, postoperative CEA levels were found to be elevated in patients with liver metastases.

The online platform used the newest web technologies and it was developed to collect data about the patients with colorectal cancer. This application was designed as a module that will be further integrated into a larger platform which intends to be a tool for the management of colorectal cancer patients from all over the country.

On the other hand, the structure of this platform brought back together two components: a backend that handles the processing of information whose code is written in PHP (HypertextPreprocessor) through a framew ork called Laravel and a frontend dealing with the user interaction

Table 2

CORRELATIONS BETWEEN POSTOPERATIVE/PREOPERATIVE CA19-9/CEA VALUES AND RECURRENCE SITE, $* n=$ no. OF PATIENTS Postoperative

\begin{tabular}{|c|c|c|c|}
\hline & $\begin{array}{l}\text { Normal postoperative } \\
\text { CA19-9 }\left({ }^{*} n=212\right)\end{array}$ & $\begin{array}{l}\text { High postoperative } \\
\text { CA19-9 }(n=45)\end{array}$ & $P$-value \\
\hline Normal preoperative CA19-9 & $183(86.32 \%)^{-}$ & $22(48.88 \%)$ & 0.001 \\
\hline High preoperative CA19-9 & $29(13.68 \%)$ & $23(51.12 \%)$ & \\
\hline \multicolumn{4}{|c|}{ Recurrence } \\
\hline & $\begin{array}{l}\text { Liver metastasis } \\
\quad(n=27)\end{array}$ & $\begin{array}{l}\text { Peritoneal recurrence } \\
\qquad(n=14)\end{array}$ & \\
\hline High postoperative CAl9-9 & $12(44.4 \%)$ & $9(64.28 \%)$ & \\
\hline High postoperative CEA & $16(59.26 \%)$ & $11(78.54 \%)$ & 0.03 \\
\hline
\end{tabular}




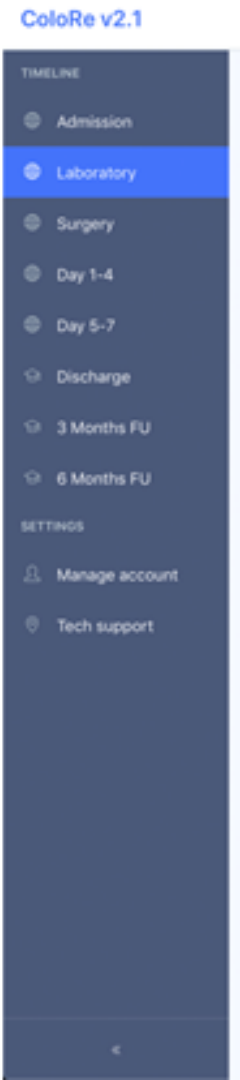

written in HTML5, CSS, and JavaScript. The JavaScript coding part was made through a framework called Vue (one of the most popular JavaScript libraries at this time).

By using this type of data acquisition application, we took the advantage of low-resource computer needs, considering that the application runs in the browser (i.e. Internet Explorer, Google Chrome, Mozilla, and Safari). Another advantage of this platform was the fact that the data processing was performed directly on the server, reducing the costs to implement projects that are based on data collection in a large number of centers. This type of application can be accessed from any device that has internet access (desktop, laptop, tablet or smart phone) making the data gathering process more accessible. Efforts have been made to make data collection as easy and as fast as possible in order to obtain a higher percentage of data completion, quality of data, and for increased compliance of the registrars. Aiming at a higher percent of data completeness, a user-friendly interface has been created with the grouping of forms in order to obtain a shorter data entry time (fig.1).

In order to ensure the best quality of the data, the form fields were divided into mandatory fields and optional ones so that if a mandatory field was not completed the data could not be submitted.

To simplify the statistical analysis, custom filters were created, and filtered data were able to be exported in excel file format. A particular issue of this project was the security of the data. In this regard, the platform included security measures like server security methods, application-level security methods (registration of the registrars with e-mail confirmation and manual access grant by a security administrator) and storing patient's personal data by anonymization. To shorten the learning curve of the application for the registrars, technical support has been provided both to the phone at a dedicated line and by email.

Data completeness in this study reached a staggering $93 \%$, withoutusing the mandatory fields option, most likely due to the ease use of the database, but possible also due to the excitement of the implementation of this new strategy for the improvement of our surgical services in colorectal cancer in our hospital.

Despite the fact that CRC incidence rates generally are higher among males versus females, at all anatomical subsites, the male-to-female incidence rate ratio increases progressively across the colon from the cecum to the rectum. The literature findings attribute CRC incidence rates of approximately $30 \%$ higher for men versus women, while mortality rates are approximately $40 \%$ higher [9]. Histologically, the majority of the cases, 194 (75.48\%) presented well or moderately differentiated cellularity. Smoking, associated pathology, location of the primary tumor was also analyzed. According to Dukes classification, more severe form of the disease was associated with an increased level of both tumor marker levels. Metastasis in liver and lung were found significantly more frequently in patients with CEA and CA 19-9 positive. Surveillance after surgery is the key component of treatment of patients with CRC. The idea of intensive postoperative surveillance is based on the idea that some of the patients can have curable recurrences [10]. An inconvenient might be the fact that CEA levels can be elevated on apparently healthy smokers and elderly individuals or in some benign conditions such as liver and gastrointestinal system inflammations of any cause [11, 12].

The tumor marker level that was elevated before the operation was found to be elevated again at the time of recurrence. The results of this study showed that $12.3 \%$ of the patients with CRC, without an increase in CEA level, had at least once an increased CA 19-9 level during the course of the disease. Of all patients $65.4 \%$ had at least once an elevation of both of the tumor markers during the course of the disease. Severity of disease, reflected by Dukes classification, and presence of metastasis were each associated with increased levels of CA 19-9 and CEA. The correlation between serum level CEA measured 
postoperative and on recurrence has been reported by other authors to be greater for liver metastases than for recurrent disease at other locations [13].

The efficacy of measuring the serum CEA and CA19-9 levels to detect or exclude a recurrence of CRC after surgery have been reported before [14, 15]. Though, it is not a standard that both tumor markers must be increase in the same time; sometimes only one of the two tumor markers increase independently.

This innovative platform was also designed as a part of intensive postoperative follow-up strategy. This strategy involves the following: a clinical examination every 3 to 6 months in the first 3 years, and every 6 months in the 4th and 5th year after surgery; Serum carcinoembryonic antigen level at every periodic medical exam for the first 3 years; Computed tomography (CT) of the chest, abdomen, and pelvis for at least 3 years; Colonoscopy at 1 year and consequently at 3 to 5 years intervals; Flexible proctosigmoidoscopy every 6 months for 3 to 5 years in patients with rectal cancers and low anterior resection who have not received pelvic irradiation. We strongly considered that the results from prospective clinical trials will remain the backbone of evidence-based medicine. One of the most important concerns was that the majority of cancer patients do not participate in clinical trials for various reasons. There are numerous advantages for using this modern concept of data centralization. Nowadays, the majority of clinical trials are initiated using electronic data capture (ECD) software. For this reason, we integrated an EDC system that is generally used with a mobile data collection system that has been widely used in healthcare programs in low-resource settings [16].

Furthermore, this platform was designed to serve as a continuous infrastructure for a large variety of research purposes. This includes prognostic research, individualized therapies in a target population according to the cohort multiple randomized controlled trial design, healthcare strategy and cost-effectiveness studies.

\section{Conclusions}

CA 19-9 can be used as additional marker to follow the disease process in patients with colorectal cancer. Both CEA and CA 19-9 are important prognostic factors for patients with colorectal cancer. High serum levels of both tumor markers are correlated with an increased disease stage and a poor long-term prognosis. The importance of this platform is that it opens a new perspective regarding the management of the cases with colorectal tumoral pathology. This study will further provide the necessary infrastructure, geared towards efficient, safe and comprehensive clinical evaluation of CRC cases.

\section{References}

1.FERLAY, J., SOERJ OMATARAM, I., DIKSHIT, R., ESER, S., MATHERS, C., REBELO, M., PARKIN, DM., FORMAN, D., BRAY, F. GLOBOCAN 2012 v1.0, Cancer Incidence and Mortality Worldwide: IARC CancerBase No. 11 [Internet] Lyon, France: International Agency for Research on Cancer, 2013.
2.FORCE, USPST, BIBBINS-DOMINGO, K., GROSSMAN, DC., CURRY, $S$ S., et al. Screening for colorectal cancer: US preventive services task force recommendation statement. JAMA, 315, 2016, p. 2564-2575.

3.FERLAY, J., SOERJ OMATARAM, I., DIKSHIT, R., et al. Cancer incidence and mortality worldwide: sources, methods and major patterns in GLOBOCAN 2012. Int. J. Cancer, 136, 2015, p. E359-E386. 4.SIEGEL, RL., MILLER, KD., JEMAL, A. Cancer statistics, 2016. CA Cancer J. Clin., 66, 2016, p. 7-30.

5.SIEGEL, RL., MILLER, KD., FEDEWA, SA., et al. Colorectal cancer statistics. CA Cancer J. Clin. 67, 2017, p.177193.

6.DUFFY, MJ ., LAMERZ, R., HAGLUND, C., NICOLINI, A., KALOUSOVA, M., HOLUBEC, L., et al. Tumor markers in colorectal cancer, gastric cancer and gastrointestinal stromal cancers: European group on tumor markers 2014 guidelines update. International journal of cancer, Journal international du cancer. 134, 11, 2014, p. 2513-22.

7.STIKSMA, J., GROOTENDORST, DC., VAN DER LINDEN, PW. CA 19-9 as a marker in addition to CEA to monitor colorectal cancer. Clinical colorectal cancer. 13, 4, 2014, p. 239-44.

8.FAUCI, AS., KASSPER, DL., LONGO, DL., BRAUNWALD, E., HAUSER, SL., JAMESON, J L., et al. Harrison's Principle of Internal Medicine. 17th edition. New York (NY), USA: McGraw-Hill, Medical Pub. Division, 2008.

9.MURPHY, G., DEVESA, SS., CROSS, AJ ., INSKIP, PD., MCGLYNN, KA., COOK, MB. Sex disparities in colorectal cancer incidence by anatomic subsite, race and age. Int. J. Cancer, 128, 2011, p. 1668-1675.

10.GODHI, S., GODHI, A., BHAT, R., SALUJA, S. Colorectal Cancer: Postoperative Follow-up and Surveillance. Indian J ournal of Surgery. 79, 3, 2017, p. 234-7.

11.STEVENS, D., MACKAY, I., GROUP, BP. Increased carcinoembryonic antigen in heavy cigarette smokers. The Lancet, 302, 7840, 1973, p. 1238-9.

12.CARPELAN-HOLMSTRÖM, M., LOUHIMO, J., STENMAN, UH., ALFTHAN, H., HAGLUND, CC. CEA, CA 19-9 and CA 72-4 improve the diagnostic accuracy in gastrointestinal cancers. Anticancer research, 22, 4, 2002, p. 2311-6.

13.PENG, J ., CAI, SJ ., LU, HF., CAI, GX., LIAN, P., GUAN, ZO., WANG, $M H ., X U, Y$. Predicting prognosis of rectal cancer patients with total mesorectal excision using molecular markers. World J. Gastroenterol., 13, 2007, p. 3009-3015.

14.HARA, M., SATO, M., TAKAHASHI, H., TAKAYAMA, S., TAKEYAMA, H. Accuracy of monitoring serum carcinoembryonic antigen levels in postoperative stage III colorectal cancer patients is limited to only the first postoperative year. Surg. Today, 41, 2011, p. 1357-1362.

15.KAWAMURA, YJ ., TOKUMITSU, A., MIZOKAMI, K., SASAKI, J., TSUJ INAKA, S., KONISHI, F. First alert for recurrence during followup after potentially curative resection for colorectal carcinoma: CA 19-9 should be included in surveillance programs. Clin. Colorectal Cancer, 9, 2010, p. 48-51.

16.SURKA. S., EDIRIPPULIGE, S., STEYN, K., et al. Evaluating the use of mobile phone technology to enhance cardiovascular disease screening by community health workers. Int. J. Med. Inform., 83, 2014, p. 648-54.

$\overline{\text { Manuscript received: } 21.08 .2018}$ 